\title{
A study of top-quark mass measurement using the lepton energy distribution at the Large Hadron Collider
}

\author{
Seo Hyun An ${ }^{1}$, Sayaka Kawabata ${ }^{2}$, Tae Jeong Kim ${ }^{1, a}$ \\ ${ }^{1}$ Department of Physics, Hanyang University, Seoul 04764, South Korea \\ ${ }^{2}$ Institute of Convergence Fundamental Studies, Seoul National Universiy of Science and Technology, Seoul 01811, South Korea
}

Received: 14 April 2017 / Accepted: 12 December 2017 / Published online: 19 December 2017

(C) The Author(s) 2017. This article is an open access publication

\begin{abstract}
We present a feasibility study of top-quark mass measurement using the lepton energy distribution in the topquark decay $t \rightarrow b W \rightarrow b \ell v$ at the LHC. The method used in this study requires only the lepton energy distribution at parton level. The analysis is performed in the lepton + jets final state by using fast simulation data corresponding to an integrated luminosity of approximately $20 \mathrm{fb}^{-1}$ at $\sqrt{s}$ $=14 \mathrm{TeV}$. Events with exactly one lepton, at least four jets and two $b$ jets are selected. The lepton energy distribution at parton level is obtained by applying the bin-by-bin unfolding technique. The study shows that the pole mass of the top quark can be measured with an uncertainty of the order of 1 $\mathrm{GeV}$.
\end{abstract}

\section{Introduction}

The top quark, which has the largest mass in the Standard Model (SM), is believed to give us a hint for new physics due to its inevitable large coupling with the Higgs boson discovered in 2012 at the Large Hadron Collider (LHC). The top-quark mass value is one of key issues of the current researches. In particular, it plays an important role in testing the stability of the SM Higgs potential. The most precise measurement of the top-quark mass is $172.44 \pm 0.13$ (stat.) \pm 0.47 (syst.) $\mathrm{GeV}$ from the published CMS result [1] at the LHC. The result is limited by the systematic uncertainty, coming mainly from the modeling of the hadronization. The ATLAS collaboration has also measured the top-quark mass of 172.84 \pm 0.34 (stat.) \pm 0.61 (syst.) $\mathrm{GeV}$, which is mostly limited by the systematic uncertainty from the jet energy scale [2].

These measured top-quark masses are believed to be different from the pole mass due to non-perturbative effects

\footnotetext{
a e-mail: taekim@hanyang.ac.kr
}

like hadronization. To date, the pole mass has been measured with a relatively large uncertainty of around $2 \mathrm{GeV}$ from the $t \bar{t}$ cross section measurements $[3,4]$ and using $t \bar{t}+1$ jet events [5]. In order to reduce the uncertainty, different approaches are to be explored. One of such approaches is in the direction of utilizing leptonic observables [6-8]. Since leptons do not involve QCD activities, leptonic observables are advantageous to the extraction of the top-quark pole mass. The latest measurement using lepton differential distributions [9] has an uncertainty as close as $1.5 \mathrm{GeV}$.

Among the approaches utilizing leptonic observables, the method proposed in Ref. [8] has an unique feature. The observable required to this method is only the lepton energy distribution in the top-quark decay in the laboratory frame, while the theoretical prediction "compared" with it is just the lepton energy distribution in the top-quark rest frame. In other words, this method has a boost-invariant nature and is independent of top-quark velocities. This method is called "weight function method" as it uses a characteristic weight function $W\left(E_{\ell}, m\right)$. The essence of the method is as follows: with the lepton energy distribution $D\left(E_{\ell}\right)$ from an experiment, there are infinite number of weight functions for which the following quantity,

$I(m)=\int d E_{\ell} D\left(E_{\ell}\right) W\left(E_{\ell}, m\right)$,

vanishes when the parameter $m$ is equal to the true mass value of the top quark, i.e. $I\left(m=m_{t}^{\text {true }}\right)=0$. For the detail of the method, see Refs. $[10,11]$, which originally proposed the weight function method for the mass reconstruction of the Higgs boson.

A practical defect of this method is the fact that it requires the whole energy distribution at parton level which includes the region outside the detector acceptance. In the LHC experiments, events with low-energy leptons are not available due to a lepton $p_{T}$ trigger. In Ref. [8], this problem was coped 
with by compensating the low energy part of the distribution with Monte-Carlo (MC) events. Since the compensating MC part has $m_{t}$ dependence, a way to independently extract the top-quark mass was invented. In addition, in order to avoid uncertainties related to the MC events, a way of determining the normalization of the compensating part was devised. Furthermore, an event selection was carefully chosen not to deform the parton-level distribution of the remaining part. Although these devices work well, they induce additional complexity.

In this paper, we perform a feasibility study of the topquark mass measurement using the weight function method at the LHC. In contrast to the study in Ref. [8], we use an unfolding technique to obtain the parton-level distribution, which would make this method simpler and handier experimentally. The lepton energy distribution at parton level is obtained by a simple bin-by-bin unfolding for the lepton+jets decay channel. We adopt more realistic event selection and detector simulation than the study in Ref. [8]. We also estimate some of the major uncertainties that are expected in realistic data analyses at the LHC. We show that this method can provide an independent verification of the top-quark mass measurement and provide pointers toward possible improvements with Run 2 data at the LHC.

\section{Samples}

Simulated $p p$ collision data samples for the $t \bar{t}$ process are generated at a center-of-mass energy of $14 \mathrm{TeV}$, by using MadGraph5 (v2.4.0) [12] at the leading order due to a limit of computer resources, and interfaced with PYTHIA (v6.428) [13] for parton showering and hadronization. The $t \bar{t}$ samples are generated for five different values of the topquark mass, 167, 170, 173, 176 and $179 \mathrm{GeV}$. For each signal sample, $600 \mathrm{~K}$ events are generated. A sample with a topquark mass of $173 \mathrm{GeV}$ is generated separately to obtain a detector response correction required in the unfolding procedure. The sample for the unfolding is statistically independent of the signal data sample with the size of $1200 \mathrm{~K}$ to avoid any statistical bias.

To emulate a detector performance, the generated events are processed through the DELPHES package (v3.3.2) [14] using the public CMS detector card. Similar to the CMS reconstruction, the objects from the particle-flow algorithm implemented in DELPHES are used throughout this analysis.

Pileup events are not simulated in this analysis. Although the effects of pileup can be merged with the simulated events in the DELPHES package, pileup mitigation, which will be developed at the LHC experiments, can reduce the pileup effects significantly. It is also important to understand the physics without pileup events. Therefore, we focus on the physics under the condition that there are no pileup effects.
In the DELPHES fast simulation, momenta of all the physics objects such as electrons, muons and jets, are smeared as a function of their transverse momenta $\left(p_{\mathrm{T}}\right)$ and pseudorapidities $(\eta)$ so that the detector effects in the CMS experiment are simulated. Reconstruction efficiencies of electrons, muons and jets are also parameterized as functions of $p_{\mathrm{T}}$ and $\eta$ based on the public information from the CMS experiment.

The muon identification efficiency is set to $95 \%$ for $p_{\mathrm{T}}>$ $10 \mathrm{GeV}$ and $|\eta|<2.4$. The electron identification efficiency is set to $95 \%$ for $p_{\mathrm{T}}>10 \mathrm{GeV}$ and $|\eta|<1.5$, and $85 \%$ for $p_{\mathrm{T}}$ $>10 \mathrm{GeV}$ and $1.5<|\eta|<2.5$. Isolated muons and electrons are selected by applying a relative isolation of $I_{\text {rel }}<0.1$, where $I_{\text {rel }}$ is defined as the sum of the surrounding energy of the particle-flow tracks, photons and neutral hadrons divided by $p_{\mathrm{T}}$ of the muon or electron.

The particle-flow jets used in this analysis are clustered by using particle-flow tracks and particle-flow towers. If a jet is already reconstructed as an isolated electron, muon or photon, this jet is excluded from further consideration. The $b$-tagging efficiency parameterized as a function of $p_{\mathrm{T}}$ and $\eta$ ranges from 20 to $50 \%$. The fake $b$-tagging rate for light-flavor jets is set to $0.1 \%$, which corresponds to the tight working point in the CMS paper Ref. [15].

\section{Event selection}

Events are selected based on the decay topology of the topquark pair in the lepton+jets channel. The event should have exactly one isolated lepton $(e, \mu)$ with $p_{\mathrm{T}}>20 \mathrm{GeV}$ and $|\eta|<$ 2.1. Events are further selected by requiring at least four jets with $p_{\mathrm{T}}>30 \mathrm{GeV}$ and two $b$-tagged jets to reject SM backgrounds such as $W+$ jets and single-top events. The acceptance after all the requirements is $4.3 \%$. After this typical event selection for the lepton+jets channel, the background contribution is expected to be less than $10 \%$ level [16]. In the unfolding procedure which will be described in Sect. 4, the remaining background after the selection is assumed to be subtracted from data. A possible background contribution and its uncertainty are not considered in this analysis.

\section{Measurement}

The weight function method requires the lepton energy distribution at parton level. In order to obtain the parton-level distribution, we use an unfolding technique [17] for removing effects of detector performance, photon radiation, lepton isolation, the event selection, etc. The lepton energy distribution at reconstruction level is unfolded back to the parton-level distribution by using a simple bin-by-bin unfolding. For the unfolding, the additional sample with the top- 


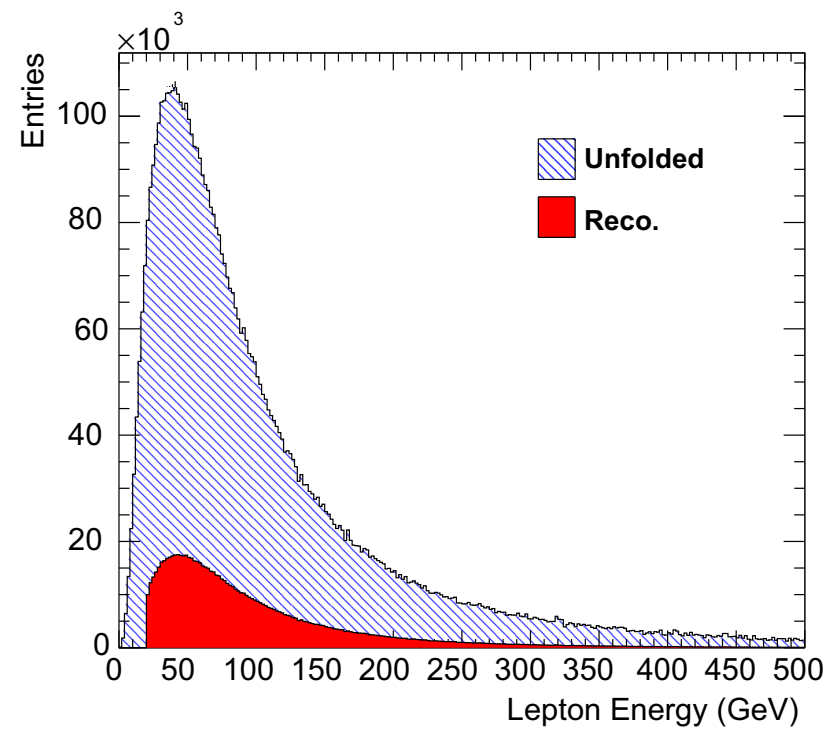

Fig. 1 Energy distribution of lepton at reconstruction level for the topquark mass of $173 \mathrm{GeV}$ (red color) and the unfolded distribution at parton level (blue color). The energy distribution at reconstruction level is unfolded using an additional sample with the top quark mass of 173 $\mathrm{GeV}$

quark mass of $173 \mathrm{GeV}$ is used. The lepton energy distribution at reconstruction level and the unfolded distribution are shown in Fig. 1. A bin width of $2 \mathrm{GeV}$ is used for the lepton energy distributions. In reality, a more complicated unfolding such as regularization might be required to correct effects of detector resolution and bin migration, which can arise from energy loss due to final-state radiation from a muon or Bremsstrahlung from an electon.

In this analysis, the unfolding is done in two steps. The first step is to correct the event selection effect. The energy distribution after the final selection is unfolded back to the distribution at preselection level with the single-lepton requirement. In this first step, since events are within the acceptance range, a data-driven method could be used. The second step is to correct detector effects such as acceptance and resolution on the lepton energy spectrum in order to obtain the energy distribution at parton level from the distribution at preselection level with the single-lepton requirement. In this second step, the response sample with the top-quark mass of $173 \mathrm{GeV}$ is used. In the unfolding procedure, it is important to have a statistically independent sample to avoid any bias. Therefore, an additional $1200 \mathrm{~K}$ events are generated for the response distribution.

At reconstruction level, there are no events in a low-energy region below $20 \mathrm{GeV}$ due to the lepton-trigger requirement of $p_{\mathrm{T}}>20 \mathrm{GeV}$. Therefore, we rely on the MC simulation below the $p_{\mathrm{T}}$ threshold of $20 \mathrm{GeV}$. Figure 2 shows unfolded distributions at the top-quark masses of 167, 173 and 179 $\mathrm{GeV}$. The distribution below the $20 \mathrm{GeV}$ threshold at parton level is from the response sample generated at $m_{t}=173 \mathrm{GeV}$.

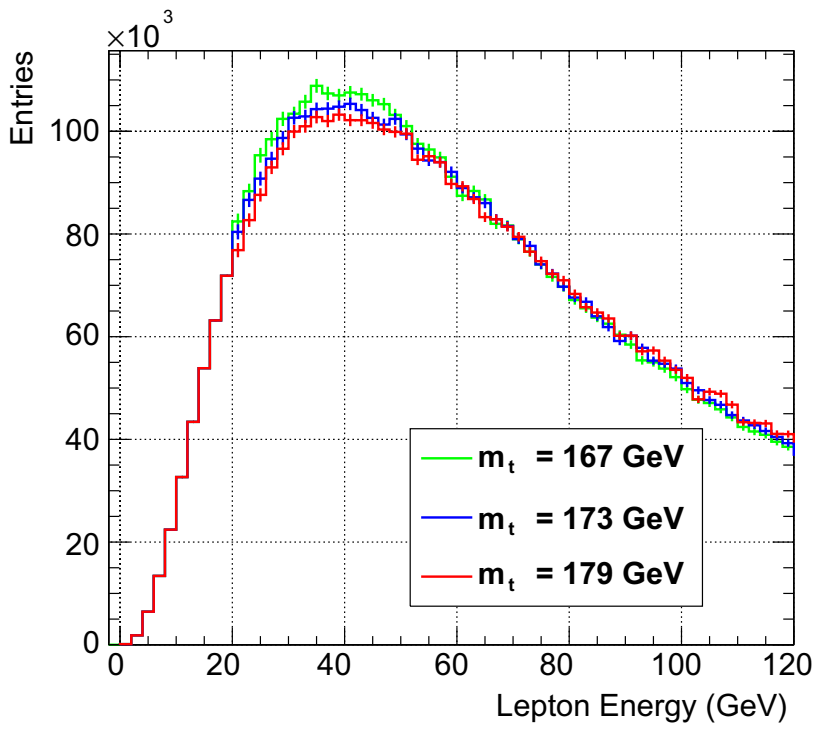

Fig. 2 Unfolded energy distributions of lepton at parton level at the top-quark masses of 167,173 and $179 \mathrm{GeV}$

After the unfolding procedure, the unfolded energy distribution at parton level is used in the weight function method. The weight functions $W\left(E_{\ell}, m\right)$ used in this analysis are provided by authors of Ref. [8]. The explicit form of the weight function is

$W\left(E_{\ell}, m\right) \propto \int d E \mathcal{D}_{0}(E ; m) \frac{1}{E E_{\ell}} \frac{\left(E_{\ell} / E\right)^{n}-\left(E / E_{\ell}\right)^{n}}{\left[\left(E_{\ell} / E\right)^{n}+\left(E / E_{\ell}\right)^{n}\right]^{2}}$,

where $\mathcal{D}_{0}(E ; m)$ is a theoretical prediction at leading-order for the distribution of lepton energy $E$, calculated in the topquark rest frame with a top-quark mass value $m$. The weight functions corresponding to $n=2,3,5,15$ for $m=173 \mathrm{GeV}$ are shown in Fig. 3.

With these weight functions, the following method is applied. As explained in Sect. 1, we calculate weighted integrals of the lepton energy distribution [Eq. (1)]. With binned data, the integral in Eq. (1) is replaced by a sum:

$I(m)=\sum_{i} N_{i} W\left(E_{i}, m\right) / \sum_{j} N_{j}$

where $N_{i}$ and $E_{i}$ are the number of entries and the lepton energy, respectively, for $i$-th bin of the lepton energy distribution. Then the reconstructed top-quark mass $m_{t}^{r e c}$ is extracted through $I\left(m=m_{t}^{r e c}\right)=0$. We multiply the unfolded energy distribution and the weight function bin by bin, and obtain the weighted sum.

Figure 4 shows the weighted sums over the parton-level energy distribution with the weight functions corresponding to $n=2,3,5,15$. The zeros of the weighted sums indicate 


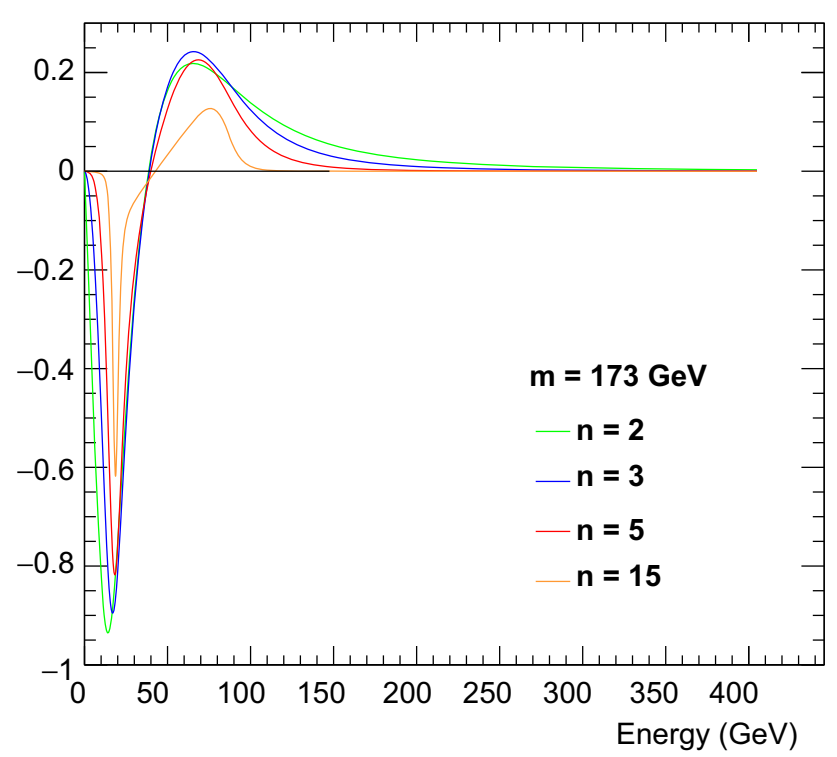

Fig. 3 Weight functions $W\left(E_{\ell}, m\right)$ provided by the authors of Ref. [8] corresponding to $n=2,3,5,15$ in Eq. (2) with $m=173 \mathrm{GeV}$

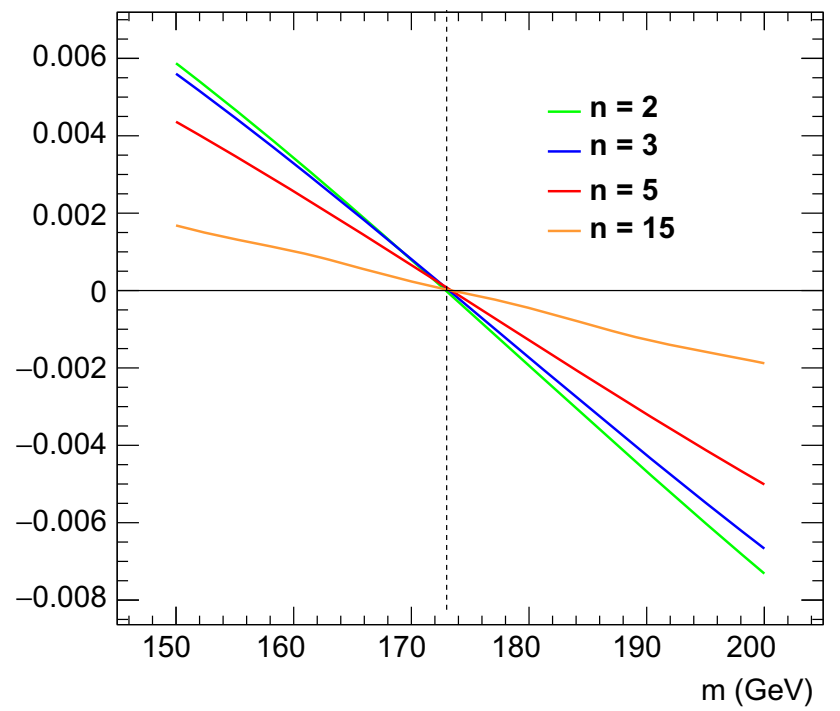

Fig. 4 Weighted sums $I(m)$ over the unfolded lepton energy distribution with the weight functions corresponding to $n=2,3,5,15$. The input value of the top-quark mass is $173 \mathrm{GeV}$, as its position is indicated by a dashed line. The horizontal axis is the mass parameter $m$ used in the calculation of $W\left(E_{\ell}, m\right)$. The top-quark mass is reconstructed from $I\left(m=m_{t}^{r e c}\right)=0$

reconstructed top-quark masses. In this figure, the input topquark mass to the data sample is set to $173 \mathrm{GeV}$. The plot shows that the input value is correctly reconstructed for each $n$ using the unfolded energy distribution.

\section{Results}

Figure 5 shows reconstructed top-quark masses versus input masses in a $3-\mathrm{GeV}$ step for the $n=2$ weight function. The

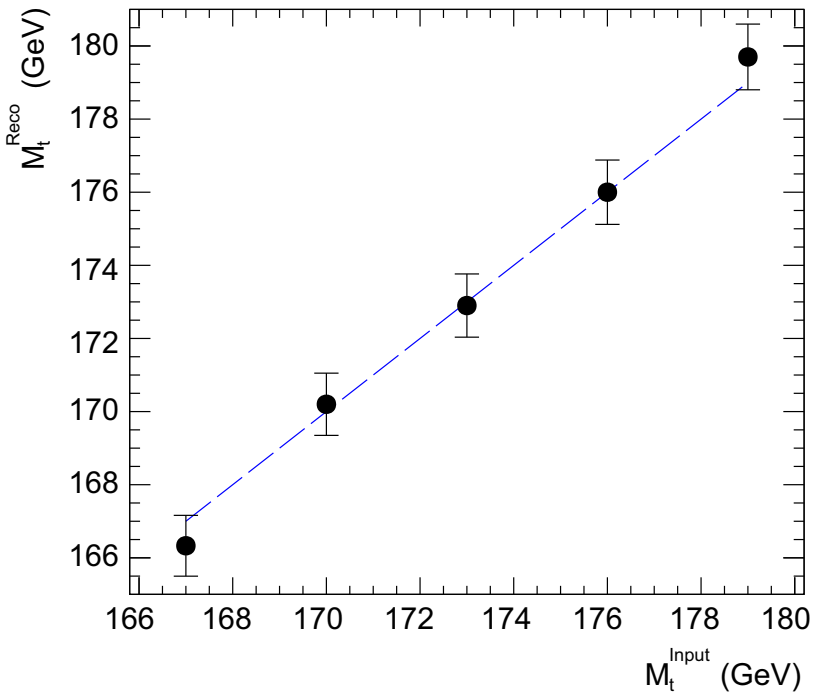

Fig. 5 Validation with different input values of the top-quark mass using the response distribution with $m_{t}=173 \mathrm{GeV}$. The vertical axis indicates the reconstructed top-quark mass and the horizontal axis indicates the input mass. The dashed line indicates the ideal case where the input mass is the same as the reconstructed mass. The statistical uncertainty is shown together with the central value

Table 1 Input and reconstructed masses using the response distribution with $m_{t}=173 \mathrm{GeV}$. The statistical uncertainty for each mass value is $0.5 \%$

\begin{tabular}{lccccc}
\hline Input $\mathrm{m}_{t}(\mathrm{GeV})$ & 167 & 170 & 173 & 176 & 179 \\
\hline Reco. $\mathrm{m}_{t}(\mathrm{GeV})$ & & & & & \\
$\quad n=2$ & 166.3 & 170.2 & 172.9 & 176.0 & 179.7 \\
$n=3$ & 166.4 & 170.4 & 173.2 & 176.3 & 179.9 \\
$n=5$ & 166.3 & 170.6 & 173.4 & 176.4 & 179.9 \\
$n=15$ & 166.6 & 170.8 & 173.4 & 176.7 & 179.5 \\
\hline
\end{tabular}

response sample with the top-quark mass of $173 \mathrm{GeV}$ is used for the unfolding including the correction below the $20 \mathrm{GeV}$ threshold. The statistical uncertainty is estimated by varying each bin randomly within the statistical uncertainty in the unfolded distribution. The statistical uncertainty shown on the unfolded distribution is the sum of the statistical uncertainties from the response distribution and the input distribution. This uncertainty on the unfolding is obtained by running toy MC experiments. The estimated statistical uncertainty is around $0.5 \%$ which corresponds to about $0.8 \mathrm{GeV}$ for the topquark mass of $173 \mathrm{GeV}$. The statistical uncertainty described above is shown in Fig. 5.

Table 1 shows the input and reconstructed top-quark masses using the weight function corresponding to $n=2,3,5$, 15. The reconstructed top-quark masses are consistent with the input top-quark masses within the sizes of the statistical error. Note that the results with different $n$ involve different sizes of statistical and systematic uncertainties, although 
Table 2 Input and reconstructed masses for various lepton $p_{\mathrm{T}}$ thresholds from $20 \mathrm{GeV}$ to $26 \mathrm{GeV}$ in steps of $2 \mathrm{GeV}$. The weight function for $n=2$ is used

\begin{tabular}{rccccc}
\hline Input $\mathrm{m}_{t}(\mathrm{GeV})$ & 167 & 170 & 173 & 176 & 179 \\
\hline Reco. $\mathrm{m}_{t}(\mathrm{GeV})$ & & & & & \\
$p_{\mathrm{T}}>20 \mathrm{GeV}$ & 166.3 & 170.2 & 172.9 & 176.0 & 179.7 \\
$p_{\mathrm{T}}>22 \mathrm{GeV}$ & 167.7 & 170.3 & 173.6 & 175.5 & 178.6 \\
$p_{\mathrm{T}}>24 \mathrm{GeV}$ & 168.7 & 170.9 & 173.6 & 175.3 & 177.4 \\
$p_{\mathrm{T}}>26 \mathrm{GeV}$ & 169.8 & 171.1 & 173.3 & 174.9 & 176.2 \\
\hline
\end{tabular}

they are strongly correlated. In addition, there would be a systematic bias due to the $2-\mathrm{GeV}$ bin width. Since a weight function with larger $n$ has a sharper form in the low-energy region (see Fig. 3), the bias due to the bin width is larger for the larger- $n$ weight function.

To see effects of the lepton $p_{\mathrm{T}}$ threshold on the result, the analysis is repeated with different lepton $p_{\mathrm{T}}$ thresholds of 22 , 24 and $26 \mathrm{GeV}$. Increasing the threshold would lead to a larger bias on the reconstructed top-quark mass. Table 2 shows input and reconstructed masses for various lepton $p_{\mathrm{T}}$ thresholds. One can see in Table 2 that the reconstructed top-quark mass approaches the top-quark mass value of the response sample, namely $173 \mathrm{GeV}$, as the threshold increases. With the $20 \mathrm{GeV}$ threshold, the bias is sufficiently small compared with the statistical uncertainty.

\section{Discussion}

In this section, we discuss and estimate main systematic uncertainties that can arise in this method. The most serious bias is caused by the fact we have to rely on the response distribution from the MC sample for the lepton energy distribution below the threshold. As the lepton energy threshold goes up, we rely more and more on the response sample. In Sect. 5, we showed this possible bias is negligible when the lepton energy threshold of $20 \mathrm{GeV}$ is used. The result with this threshold is consistent with the input top-quark mass within the statistical uncertainty of $0.5 \%$. However, with a threshold of above $20 \mathrm{GeV}$, the bias becomes larger than the statistical uncertainty, and the method used in this paper has a difficulty. Therefore, it is desired that the lepton $p_{\mathrm{T}}$ threshold is as low as possible. Note that this is not a critical problem for the weight function method itself. As explained in Sect. 1, this problem is solved in Ref. [8], by imposing a consistency condition that the reconstructed mass is equal to the mass for the MC sample below the threshold. The result in our study demonstrates that the simple way used in this analysis can be applied only with a $p_{\mathrm{T}}$ trigger as low as $20 \mathrm{GeV}$. With a lepton $p_{\mathrm{T}}$ trigger above $20 \mathrm{GeV}$, it would be essential to apply a supplementary method like the one described in Ref. [8].

There would be a systematic uncertainty from the datadriven correction in the first step of the unfolding described in Sect. 4. In order to obtain the correction factor for the selection efficiency, an orthogonal event selection can be applied to enhance top-quark events. However, it would not be trivial to find such an orthogonal event selection. In this analysis, the uncertainty in this step is ignored but can be significant in a real analysis. In addition, uncertainties on the background subtraction are not included in this analysis but can contribute. The largest experimental uncertainty in the top-quark pair cross section used to determine the pole mass is from the lepton energy scale and resolution $[3,4]$.

Uncertainties from the factorization and renormalization scales are estimated by varying the scales for input distributions by a factor of two up and down with respect to their reference values for the lepton energy distribution at the topquark mass of $173 \mathrm{GeV}$. The same response sample with the nominal scale is kept for the unfolding. The uncertainty of $0.3 \%$ is assessed by taking the difference in the result. It should be noted that these scale uncertainties are the dominant ones in the estimate of uncertainties in the study of Ref. [8]. The smallness of the scale uncertainties compared to those in Ref. [8] is an advantage of using the unfolding. It would also be required to have an extensive validation of the unfolding by using different MC generators to check any possible bias from theoretical predictions. In particular, the validation with NLO generators would be very useful.

The weight function method is based on the assumption that the top quark is on-shell. Thus, the actual finite width of the top quark causes a deviation to the reconstructed mass. We estimate the size of this deviation by examining the invariant mass distribution of the top quark at parton level. With the parameter setting that the cutoff for the Breit-Wigner distribution in the configuration of the MadGraph package is at $m_{t} \pm 50 \Gamma_{t}$, the mean value of the mass distribution is shifted from the input mass value by the amount of 0.3 $\mathrm{GeV}$ for each mass. Therefore, we expect a systematic shift of the order of $0.3 \mathrm{GeV}$ in the result for the reconstructed mass. In real experiments, the effect of the top-quark width can be estimated by simulation analyses with MC generators which take into account the top-quark finite-width effects more thoroughly (see, for example, Ref. [18]).

Overall, the sum of the statistical and systematic uncertainties considered in this analysis is less than $1 \mathrm{GeV}$.

This study is performed at leading order, using the leadingorder event generator and the leading-order theoretical prediction for the lepton energy distribution $\mathcal{D}_{0}$. If we include the next-to-leading order (NLO) corrections to them in the on-shell scheme, a reconstructed mass is identified with the top-quark pole mass. More specifically, a weight function at NLO is calculated with $\mathcal{D}_{0}^{\mathrm{NLO}}\left(E ; m^{\text {pole }}\right)$, which is the NLO 
distribution of lepton energy (in the top-quark rest frame) with a top-quark pole mass $m^{\text {pole }}$. Then one can read off the NLO pole mass value from a weighted integral through $I\left(m^{\text {pole }}=m_{t}^{\text {pole, } r e c}\right)=0$. Note that for the purpose of extracting the NLO pole mass, only the corrections to the topquark decay process are required since the theoretical prediction used in the weight functions, $\mathcal{D}_{0}^{(\mathrm{N}) \mathrm{LO}}$, is the distribution in the top-quark rest frame. Thanks to the boost-invariant nature of the weight function method (as mentioned in Sect. 1 and proven in Refs. $[10,11])$, the method relies on only the top-quark decay process for theoretical prediction. Note also that the theoretical corrections to the top-quark decay process are much smaller than those to the production process, so that experimental consequences are insensitive to the corrections to the decay process. These suggest that the application of this analysis to the NLO pole-mass extraction is straightforward and the experimental uncertainties estimated in this leading-order study will not change significantly.

\section{Conclusions}

We estimated the sensitivity of the top-quark mass measurement with the weight function method by using simulation samples. This method requires only the lepton energy distribution at parton level. Events with exclusively one lepton, at least four jets and two $b$ jets are selected. The lepton energy distribution at reconstruction level after the selection is unfolded back to the energy distribution at parton level. In the region below the energy threshold of $20 \mathrm{GeV}$, the response sample with the top-quark mass of $173 \mathrm{GeV}$ is used. The reconstructed mass of the top quark with the weight function method is consistent with the input mass within the statistical uncertainty of $0.8 \mathrm{GeV}$. We discussed and estimated some of main systematic uncertainties expected in this method. Taking into account the statistical and systematic uncertainties, the estimated uncertainty of the reconstructed top-quark mass is of the order of $1 \mathrm{GeV}$. This uncertainty is compatible with the current uncertainty of $2 \mathrm{GeV}$ in the measurement of the top-quark pole mass. Therefore, the weight function method could provide an alternative approach to measure the top-quark mass without introducing large systematic uncertainties that can arise due to the jet energy measurement. This study shows that the weight function method can also provide an independent verification of the top-quark mass measurement and provide pointers toward possible improvements with Run 2 data.

Acknowledgements This work was supported by the research fund of Hanyang University (HY-2015). This work was supported by a National Research Foundation of Korea Grant funded by the Korean governments (NRF-2014R1A1A2056283 and NRF-2014S1A2A2028546). The work of S.K. was supported by Basic Science Research Program through the National Research Foundation of Korea (NRF) funded by the Ministry of Science, ICT and Future Planning (Grant No. NRF2014R1A2A1A11052687).

Open Access This article is distributed under the terms of the Creative Commons Attribution 4.0 International License (http://creativecomm ons.org/licenses/by/4.0/), which permits unrestricted use, distribution, and reproduction in any medium, provided you give appropriate credit to the original author(s) and the source, provide a link to the Creative Commons license, and indicate if changes were made.

Funded by $\mathrm{SCOAP}^{3}$.

\section{References}

1. CMS Collaboration, Measurement of the top quark mass using proton-proton collisions at $\sqrt{s}=7$ and $8 \mathrm{TeV}$. Phys. Rev. D 93, 26 (2016). https://doi.org/10.1103/PhysRevD.93.072004. arXiv:1509.04044

2. ATLAS Collaboration, Measurement of the top quark mass in the $t \bar{t} \rightarrow$ dilepton channel from $\sqrt{s}=8 \mathrm{TeV}$ ATLAS data. Phys. Lett. B 761, 350 (2016). https://doi.org/10.1016/j.physletb.2016.08.042. arXiv: 1606.02179

3. CMS Collaboration, Measurement of the $t \bar{t}$ production cross section in the $e \mu$ channel in proton-proton collisions at $\sqrt{(} s)$ $=7$ and 8 TeV. JHEP 08, 029 (2016). https://doi.org/10.1007/ JHEP08(2016)029. arXiv:1603.02303

4. ATLAS Collaboration, Measurement of the $t \bar{t}$ productio $\mathrm{n}$ cross section in the $e \mu$ events with b-tagged jets in pp collisions at $\sqrt{(s)}=7$ and $8 \mathrm{TeV}$ with the ATLAS detector. Eur. Phys. J. C 74, 3109 (2014). https://doi.org/10.1140/epjc/ s10052-014-3109-7. arXiv:1406.5375

5. ATLAS Collaboration, Determination of the top-quark pole mass using $t \bar{t}+1$-jet events collected with the ATLAS experiment in 7 TeV pp collisions. arXiv:1507.01769

6. CDF Collaboration, Measurement of the Top Quark Mass in the Lepton+Jets Channel Using the Lepton Transverse Momentum. Phys. Lett. B 698, 371 (2011). https://doi.org/10.1016/j.physletb. 2011.03.041. arXiv:1101.4926

7. S. Frixione, A. Mitov, Determination of the top quark mass from leptonic observables. JHEP 09, 012 (2014). https://doi.org/10. 1007/JHEP09(2014)012. arXiv:1407.2763

8. S. Kawabata, Y. Shimizu, Y. Sumino, H. Yokoya, Weight function method for precise determination of top quark mass at Large Hadron Collider. Phys. Lett. B 741, 232 (2014). https://doi.org/10. 1016/j.physletb.2014.12.044. arXiv:1405.2395

9. ATLAS Collaboration, Measurement of lepton differential distributions and the top quark mass in $t \bar{t}$ production in pp collisions at $\sqrt{s}=8 \mathrm{TeV}$ with the ATLAS detector. arXiv:1709.09407

10. S. Kawabata, Y. Shimizu, Y. Sumino, H. Yokoya, Boost-Invariant Leptonic Observables and Reconstruction of Parent Particle Mass. Phys. Lett. B 710, 658-664 (2012). https://doi.org/10.1016/j. physletb.2012.03.050. arXiv:1107.4460

11. S. Kawabata, Y. Shimizu, Y. Sumino, H. Yokoya, Measurement of physical parameters with a weight function method and its application to the Higgs boson mass reconstruction. JHEP 08, 129 (2013). https://doi.org/10.1007/JHEP08(2013)129. arXiv:1305.6150

12. J. Alwall et al., The automated computation of tree-level and nextto-leading order differential cross sections, and their matching to parton shower simulations. JHEP 07, 079 (2014). https://doi.org/ 10.1007/JHEP07(2014)079. arXiv:1405.0301

13. T. Sjöstrand, S. Mrenna, P. Skands, PYTHIA 6.4 physics and manual. JHEP 05, 026 (2006). https://doi.org/10.1088/1126-6708/ 2006/05/026. arXiv:hep-ph/0603175

14. J. de Favereau et al., DELPHES 3, A modular framework for fast simulation of a generic collider experiment (2013). 
JHEP 02, 057 (2014). https://doi.org/10.1007/JHEP02(2014)057. arXiv: 1307.6346

15. CMS Collaboration, Identification of b-quark jets with the CMS experiment. J. Instrum. 8, P04013 (2013). https://doi.org/10.1088/ 1748-0221/8/04/P04013. arXiv:1211.4462

16. CMS Collaboration, Measurement of differential $t \bar{t}$ production cross sections in lepton + jets final states at $13 \mathrm{TeV}$. arXiv:1610.04191
17. T. Adye, Unfolding algorithms and tests using RooUnfold (2011). arXiv: 1105.1160

18. T. Jezo, J.M. Lindert, P. Nason, C. Oleari, S. Pozzorini, An NLO+PS generator for $t \bar{t}$ and $W t$ production and decay including non-resonant and interference effects. Eur. Phys. J. C 76, 691 (2016). https://doi.org/10.1140/epjc/s10052-016-4538-2. arXiv: 1607.04538 